\title{
Sleep Disordered Breathing in Pregnancy: An Obstetric Anesthesiologist's Perspective
}

\author{
Alexandra S. Bullough* \\ Department of Anesthesiology, Loyola University Medical Center, Maywood, IL, 60153, USA
}

\begin{abstract}
Anesthesiologists play a pivotal role in the management of high risk obstetric patients on labor and delivery. In the past decade sleep disordered breathing (SDB) in pregnancy and its associated comorbidities of difficult airway, pregnancy related hypertensive diseases; preeclampsia, gestational diabetes and intrauterine growth restriction were recognized and investigated. Moderate to severe SDB affects 1-5\% of women of childbearing age although it is likely much higher as the majority of women with SDB remain undiagnosed. The American Society of Anesthesiologists has published obstructive sleep apnea (OSA) practice guidelines for the general surgical population but not the obstetric patient and there is no discussion of this condition in the updated ASA practice guidelines for obstetric anesthesia. Also, validated OSA questionnaires used to screen for OSA in the general population are not appropriate tools to use in high risk parturients. This article discusses how to recognize and optimize clinical anesthetic management of the high risk parturient diagnosed with SDB. It also considers some of the unique physiological changes of pregnancy that impact the parturient with SDB and questionably the fetus.
\end{abstract}

Keywords: Sleep disordered breathing, anesthesia, pregnancy, difficult airway, preeclampsia, obesity.

\section{INTRODUCTION}

Anesthesiologists play a pivotal role in the management of high risk obstetric patients. Awareness of sleep disordered breathing (SDB) within anesthesiology circles is improving daily but it still remains undiagnosed especially in the pregnant population. This article will discuss the importance of SDB in pregnancy for anesthesiologists as well as improve their recognition, understanding and peri-operative management of SDB in pregnancy.

SDB is a common, transient complaint in pregnancy. It describes respiratory abnormalities during sleep which range from habitual snoring to obstructive sleep apnea. It is characterized by partial or complete upper airway obstruction during sleep and gives rise to hypoxemia, sleep fragmentation and disruption of normal ventilation as well as being associated with pregnancy induced hypertension and maternal obesity.

It is estimated that moderate to severe SDB affects 1-5\% [1-3] of women of childbearing age although it is likely much higher as the majority of women with SDB remain undiagnosed [1]. It is only in the past decade that SDB in pregnancy and its associated comorbidities of difficult airway, gestational hypertension, preeclampsia, gestational diabetes and intrauterine growth restriction (IUGR) were recognized and investigated [4-7]. Yet sleep apnea in pregnancy was being described as a new syndrome as early as 1978 [7].

The American Society of Anesthesiologists (ASA) 2006 practice guidelines for the peri-operative management of

*Address correspondence to this author at the Department of Anesthesiology, Loyola University Medical Center, Maywood, IL, 60153, USA; Tel: 708216 3619; Fax: 7082168941 Email: bulloughas@gmail.com patients with obstructive sleep apnea (OSA) fail to mention the obstetric patient [8] and new studies, using highly sensitive, validated OSA screening tools e.g. STOP, STOPBang questionnaires [9] have already modified the outdated guidelines in other subspecialty areas of anesthesia such as Ambulatory anesthesia [10].

Like the associated co-morbidities of pregnancy, such as, difficult airway and pre-eclampsia, pregnant women, pregnant women with SDB may also be at risk of unreported, peri-operative respiratory and cardiovascular sequelae from general anesthesia, sedation and analgesics. Nearly one third of women undergo cesarean section in the US. This operation is usually performed under neuraxial anesthesia with local anesthetic agents and opioid based analgesics. Neuraxial anesthesia is the preferred modality over general anesthesia yet it sometimes fails and in some patients, it is contraindicated as shown in Table $\mathbf{1}$.

In 2001, Bucklin et al. indicated that urgent/emergent cesarean deliveries in the United States had a 15-30\% incidence of general anesthesia [11]. This is consistent with data from the UK, with the 2000-2002 Confidential Enquiry into Maternal and Child Health (CEMACH) report suggesting general anesthesia is provided approximately $20 \%$ of the time for cesarean sections [12]. General anesthesia is also administered in non-obstetric surgery in pregnancy, most commonly for appendectomy or cholecystectomy. Nonobstetric surgery is estimated to be $0.12 \%$ which translates to 8000 cases in the US every year [13].

\section{RECOGNITION AND DIAGNOSIS OF SDB IN PREGNANCY}

Unless there is a prior diagnosis of SDB, in pregnancy, SDB appears to develop predominantly from the $2^{\text {nd }}$ 
Table 1. Maternal Contraindications to Neuraxial Anesthesia

\begin{tabular}{|l|}
\hline Significant coagulopathy (Acquired, Induced, Genetic) \\
\hline Massive hemorrhage and/or hemodynamic instability \\
\hline Maternal spinal pathology \\
\hline True allergy to local anesthetics \\
\hline Infection at site of injection \\
\hline Sepsis \\
\hline Primary herpes infection \\
\hline Increased intra-cranial pressure \\
\hline Significant maternal cardiac disease \\
\hline Significant maternal cardiac disease \\
\hline Uncooperative patient \\
\hline
\end{tabular}

trimester onwards in association with habitual snoring [14, 15]. There are no epidemiological studies to date that have determined SDB in the obstetric population undergoing anesthesia. Preoperative management of SDB in pregnancy requires (1) identification of the condition and (2) optimization of SDB and other associated comorbidities e.g. pregnancy related hypertension, preeclampsia, obesity during pregnancy and diabetes.

\section{Pre-operative Screening Questionnaires}

Updated practice guidelines for obstetric anesthesia [16] recommend conducting a focused history and physical examination before providing anesthesia care and state that although comparative studies are insufficient to evaluate the peripartum impact of this practice, literature reports of clinical characteristics maybe associated with obstetric complications. Preeclampsia, pregnancy related hypertensive disorders, obesity and diabetes are listed as such complications.

The ASA taskforce also developed a sixteen item checklist divided into three categories, comprising predisposing physical characteristics of SDB, history of airway obstruction at sleep and somnolence, to aid in the peri-operative identification and management of patients with obstructive sleep apnea. The ASA checklist demonstrated a moderate to high level of sensitivity for OSA screening when used in general surgical patients [17]. It was also able to identify patients most likely to develop perioperative complications. Yet, while modifications in the ASA guidelines have already taken place, recognition and anesthesia management of SDB in the obstetric workplace are lacking.

A 2009 meta-analysis of clinical screening tests for obstructive sleep apnea for anesthesiologists [18] and that the two most accurate questionnaires were the Berlin and Sleep Disorder questionnaires and the two most accurate clinical models were morphometry and combined clinicalcephalometry. However, it concluded due to the significant differences in study patients and surgical patients further validation of these screening tests is essential to identify the most accurate method of screening for OSA relevant to each surgical group. Again, no SDB questionnaire to date has been developed specifically for a pregnant population, and whereas the Berlin questionnaire and ASA Checklist are validated in general surgical patients [17], this validation does not transfer to the obstetric population. Furthermore, the Berlin questionnaire and the Epworth Sleepiness Scale (ESS) questionnaire have been determined as inappropriate tools to screen sleep apnea in high risk pregnant women [19].

\section{Polysomnography}

If a physician has a high suspicion for SDB in pregnancy, in order to determine the diagnosis and severity, a formal gold standard polysomnogram (PSG) should be performed and non-invasive, nasal Continuous Positive Airway pressure (CPAP) instituted if indicated. However, polysomnography is a costly, time consuming investigation that requires an overnight stay in a sleep laboratory with technician support. Appointments for an overnight PSG investigation may take weeks to months when a diagnosis is required sooner. The delay in diagnosis and overnight monitoring do not lend thmeselves well to pregnant women with busy work and family lives.

An alternative consideration for pregnant women is an ambulatory PSG using a wrist-worn Watch-PAT 200 device in pregnant women. The investigation may be performed in the comfort of home and the data reviewed in the sleep laboratory. O'Brien et al. [20] validated its use in 31 pregnant women in their third trimester with suspected SDB and concluded that Watch-PAT demonstrated excellent sensitivity and specificity for identification of obstructive sleep apnea in pregnancy.

Polysomnographic data determine a patient's Apnea Hypopnea Index (AHI); a count of the number of apneas and hypopneas per hour of sleep. AHI is a key measure used for OSA identification, for quantifying disease severity, and for defining disease prevalence in normal and clinical populations [21]. There is only one definition for apnea, cessation of airflow for $>10 \mathrm{~s}$. However, there is more than one definition of hypopnea. Using the recommended American Academy of Sleep Medicine (AASM) 2001 definition hypopnea scoring requires $\geq 30 \%$ reduction in nasal pressure signal excursions from baseline and associated $\geq 4 \%$ desaturation from pre-event baseline. The AASM defines mild OSA as AHI 5-15, moderate OSA as AHI 15-30 and severe OSA as AHI $>30$ [22]. A study by Kim and Lee [23] in a non-pregnant population showed that the occurrence of difficult intubation could be predicted from high AHI values; notably an $\mathrm{AHI}>40$.

Pulse oximetry measurement, also a component of PSG has in its own right also been considered as an assessment tool of OSA. The oxygen desaturation index (ODI) is determined by the number of episodes per hour of oxygen desaturation of $\geq 4 \%$ (ODI 4\%). Gyulay et al. [24] suggested that a positive oximetry test result has a high positive predictive value for OSA as the ODI detects a pattern of deoxygenation and reoxygenation that is most consistent with sleep-apnea syndromes such as SDB [25].

\section{Anesthetic History and Examination}

An anesthesiologist's ability to perform a thorough airway assessment and acquire a history focusing on 
previous anesthetic issues, sleep habits, witnessed apnea, daytime sleepiness, snoring, nasal rhinitis medications, allergies, time of last meal and current obstetric state is essential for any obstetric anesthetic assessment yet is vital prior to a pregnant patient with SDB undergoing general anesthesia. If the patient is already diagnosed with SDB, the anesthesiologist needs to ask about severity and duration and what has been the outcome in previous operations under general anesthesia.

The obstetric anesthesiologist should also consider anatomical changes in pregnancy such as progressive weight gain, breast enlargement, nasal and orophrayngeal mucosal engorgement, facial swelling and oropharyngeal deposition of adipose tissue that may create conditions that may not only result in a difficult airway but are also recognized risk factors for SDB. Clinical predictors for SDB such as neck circumference and tonsil size (especially in children) are not routinely sought by an anesthesiologist.

The anesthesiologist must also remember that the airway in pregnancy is not static and may require more than one examination while the parturient is on labor and delivery. As pregnancy progresses and especially during labor the maternal upper airway decreases in size [26]. Kodali et al. [27] carried out two studies. In study one $(n=61)$, the airway was photographed at the onset and end of labor. In study two $(n=21)$, upper airway volumes were measured using acoustic reflectometry at the onset and conclusion of labor. An increase in airway class was noted in study one and study two revealed significant decreases in oral volume and pharyngeal area. There was no correlation with duration of labor or fluids administered. Thus in the pregnant patient, airway evaluation immediately prior to administration of general anesthesia is essential.

Obesity and preeclampsia, associated co-morbidities of SDB in pregnancy should trigger SDB alarm bells in an anesthesiologist. Obesity is a hallmark of sleep disordered breathing [28-30]. It is defined as a Body Mass Index $(\mathrm{BMI})>30 \mathrm{~kg} / \mathrm{m}^{2}$, morbid obesity as BMI $>40 \mathrm{~kg} / \mathrm{m}^{2}$ and super obesity, as a BMI $>50 \mathrm{~kg} / \mathrm{m}^{2}$. Worryingly, super-obese parturients have become commonplace and are a great source of concern for an anesthesiologist. Obese patients also have a well-recognized association with a difficult airway [31, 32] as well as representing a major risk for maternal mortality [33] with failed intubation and gastric aspiration representing the major cause of death [34].

A correlation between preeclampsia and sleep disordered breathing [35] has also been confirmed with an associated narrowing of the airway and absence of nocturnal dipping in systolic blood pressure seen in preeclampsia [36, 37]. The edematous changes in severe preeclampsia are caused by endothelial dysfunction and are believed to emanate from the placenta. Edema may affect the face, neck and oropharyngeal tissues causing partial airway obstruction and anatomical distortion of the airway. Izci et al. [38] measured the parameters of the upper airway using acoustic reflectance in three groups of women: normal non-pregnant women, normal pregnant women at 36 weeks gestation and preeclamptic women. They reported a marked difference in airway caliber between the pregnant and non-pregnant groups when comparing measurements from seated to supine positions. The preeclamptic group of women had
Table 2. Airway Variables that Assist in Prediction of Intubation Difficulty

\begin{tabular}{|l|}
\hline $\begin{array}{l}\text { Assessment of dentition (normal, braces, edentulous, poor dentition, } \\
\text { dentures, other) }\end{array}$ \\
\hline Degree of mouth opening ( $\geq 3 \mathrm{cms}$ or $<3 \mathrm{cms}$ ) \\
\hline Thyromental distance ( $\geq 6 \mathrm{cms}$ or $<6 \mathrm{cms})$ \\
\hline $\begin{array}{l}\text { Cervical spine (normal, limited flexion, limited extension, limited } \\
\text { flexion/extension) }\end{array}$ \\
\hline Existing airway (yes/no) \\
\hline Neck anatomy (normal, thick/obese, mass, scar, radiation) \\
\hline $\begin{array}{l}\text { Jaw protusion (can protrude lower incisors beyond upper incisors, lower } \\
\text { incisors meet upper incisors, lower incisors won't meet upper incisors) }\end{array}$ \\
\hline Modified Mallampati scores ( III, or IV) \\
\hline $\begin{array}{l}\text { Measurement of neck circumference with the woman sitting upright, } \\
\text { looking straight ahead, at the level of the thyroid cartilage }\end{array}$
\end{tabular}

significantly reduced upper airway dimensions in all positions.

The relationship between SDB and difficult airway intubation is well recognized [39-42]. How does an obstetric anesthesiologist recognize a difficult parturient airway? A pertinent history and airway examination suffice in most cases, but no single airway test predicts a difficult intubation. Mallampati grades I-IV are routinely noted in an anesthetic examination and usually worsen as pregnancy progresses in a patient with SDB. The anesthesiologist's difficult airway assessment is determined by multiple anatomical airway variables (see Table 2) such as Mallampati grades 3 or 4 (defined as the soft and hard palate and base of the uvula being visible and only the hard palate being visible respectively), limited mouth opening $<3 \mathrm{cms}$, limited atlantooccipital joint extension, a large neck circumference $(>40 \mathrm{cms})$, reduced mandibular mobility, reduced thyromental distance $(<6 \mathrm{cms})$ and protuberant incisors. All the above variables are used to determine the difficulty of an orotracheal intubation. Individually, these airway assessments are not good indicators of a difficult airway yet when considered in combination by an experienced anesthesiologist they assist in airway evaluation.

To avoid the unanticipated, emergent, difficult airway Morgan et al. [43] stated that $87 \%$ of emergency or urgent cesarean sections can be anticipated by regular evaluation of parturients on a labor and delivery suite thus enabling neuraxial anesthesia to be implemented in a timely fashion and avoid instrumentation of the airway.

Upper airway patency is an important predictor of SDB. Maternal obesity and preeclampsia both compromise upper airway patency. However, nasal and oropharyngeal engorgement also arises due to the normal hormonal changes of pregnancy and increased vascular volume [44]. Hormonal changes in pregnancy are largely mediated by the action of progesterone and to a lesser extent, estrogen. The effect of estrogen on the respiratory system is less well defined. In pregnancy, the hormone progesterone is dominant. Progesterone mediates relaxation of the bronchial musculature by enhancing $\beta$-adrenergic activity. It is 


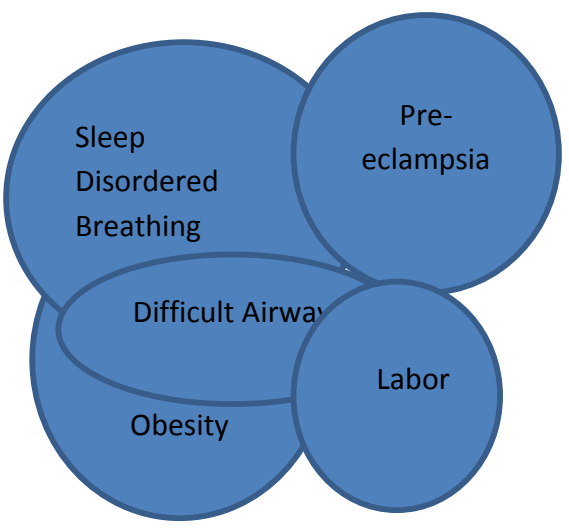

Fig. (1). Maternal causes of airway compromise.

responsible for increasing ventilation [45], which promotes development of respiratory alkalosis and hypocapnia. Progesterone levels rise from approximately $25 \mathrm{ng} / \mathrm{mL}$ at six weeks gestation to $150 \mathrm{ng} / \mathrm{mL}$ at term [46]. The high circulating level of progesterone during pregnancy increases ventilatory drive and has a potentially protective effect as the increased sensitivity of the central chemoreceptors to carbon dioxide in late pregnancy confer protection against hypoxemia and central apneas.

Estrogen promotes nasal mucosal engorgement and edema [47], as well as vasomotor rhinitis which lead to complaints of nasal stuffiness [48]. This narrowing of the upper airway increases resistance to airflow. Nasal congestion has been implicated as a risk factor for SDB in the general population [49]. Also, in the event of a general anesthetic, manipulation of the nasal passages may lead to profuse bleeding and soiling of the upper airway which not only renders oral intubation problematic but also stimulates the larynx making mask ventilation and maternal oxygenation a major challenge.

The ASA OSA practice guidelines state that a presumptive diagnosis of SDB without a PSG may be made based on the following criteria: increased BMI, increased neck circumference, habitual snoring, congenital airway abnormalities, daytime hypersomnolence, inability to visualize the soft palate and tonsillar hypertrophy and an observed apnea [8]. In association with a pertinent history, asking specifically about choking sensations during sleep, and hypertension [50], SDB in a parturient may be suspected but not confirmed. Unfortunately, no labor and delivery screening tools for SDB, as yet, have been validated in the pregnant population and the ASA task force has not yet issued any OSA practice guidelines for the pregnant patient.

\section{DRUG SIDE EFFECTS WITH GENERAL ANEST-HESIA, SEDATION AND ANALGESICS IN PREG-NANT PATIENTS WITH SDB}

Airway obstruction and hypoventilation are more likely to occur during sedation and general anesthesia (GA) i.e. depression of the central respiratory efferent pathway to the upper airway dilator muscles and upper airway reflexes which increase the collapsibility of the upper airway [51]. General anesthesia and opioid analgesics cause a dosedependent depression of upper airway muscle relaxation [51, 52].
A general anesthetic is made more challenging by the inter-relationships between maternal conditions such as moderate to severe SDB, preeclampsia and obesity that promote airway complications (Fig. 1). Benzodiazepines for anxiolysis and sedation may cause considerable relaxation of the upper airway musculature. They can reduce further an already compromised pharyngeal space which may lead to a higher risk of preoperative phases of hypopnoea and consecutive hypoxia and hypercapnia [53, 54]. Sedation plays no role in the parturient whose anesthetic status from the $2^{\text {nd }}$ trimester onwards should be considered as a "full stomach" and at high risk of pulmonary aspiration. This dangerous complication is reported in the most recent CMACE audit 2006-2008 [55] where in a fatal case a woman aspirated gastric contents on extubation upon reemergence after a cesarean section. On extubation a patient must have functional airway reflexes and be able to protect her airway. Aspiration of gastric contents was also the most common cause of death in the Fourth National Audit Project of the Royal College of Anaesthetists and the Difficult Airway Society (NAP4) report [56]. However in these cases, pulmonary aspiration occurred at induction of anesthesia or during airway instrumentation highlighting the importance of securing the airway, in high risk patients with conditions such as SDB, as rapidly as possible.

The anesthesiologist already alert to fetal concerns regarding maternal drug management needs to also heighten awareness of how analgesics, especially opioids, affect parturients with SDB in the peri-operative period. Multimodal analgesia (non-steroidal anti-inflammatory drugs and local anesthetics) should be considered wherever possible so as to avoid a narcotic load in patients already at risk of increased upper airway obstruction. Opioids may exacerbate symptoms of sleep apnea resulting in respiratory depression and respiratory arrest if administered intramuscularly, epidurally or via patient controlled analgesia [57]. Fortunately, opioids have a good safety record in pregnancy with only a handful of maternal respiratory depression case reports over the past 2 decades [58-60].

In a 2008 retrospective chart review study by Kato et al. [61], five women $(0.26 \%)$ out of 1900 who had received $0.15 \mathrm{mg}$ intrathecal morphine post cesarean section, developed bradypnea ( $\mathrm{RR}<10 / \mathrm{min})$. The incidence of severe bradypnea requiring naloxone was $1 / 1915 \quad(0.052 \%)$. However, the ever changing demographics of pregnant women, >35yr-old, obesity and more complex medical conditions may change this picture in the future.

The 2009 practice guidelines for the prevention, detection and management of respiratory depression associated with neuraxial opioid administration [62] recommend that patients with a history of sleep apnea treated with nasal CPAP be encouraged to bring in their equipment to the hospital and that all patients receiving neuraxial opioids be monitored for adequacy of ventilation, oxygenation and level of consciousness.

\section{POST-OPERATIVE IMPLICATIONS OF SDB AND GENERAL ANESTHESIA}

There is a paucity of data looking at post-operative implications of SDB in the obstetric population, however in 
the general surgical population reported complications include, respiratory events such as bradypnea, apnea, desaturations (pulse oximetry $<90 \%$ ), cardiac dysrhythmias and uncontrolled hypertension [63].

One study investigating the association of SDB with post-operative complications [64] used clinical features of SDB and nocturnal pulse oximetry as a surrogate marker for SDB. An ODI $\geq 4 \%$ (ODI $4 \%$ ) was determined. Subjects with five or more desaturations per hour were compared to subjects with less than five desaturations per hour. Complications were greater in the group with an ODI $4 \% \geq 5$ and included hypoxemia, atelectasis, dysrhythmias and chest pain.

Post-operative management of high risk parturients needs to be addressed. Pregnant patients diagnosed with sleep disordered breathing warrant intensive short-term monitoring post-operatively. The ASA guidelines [8] recommend in non-obstetric patients with obstructive sleep apnea continuous post-operative pulse oximetry in a step down unit with appropriate nursing care, lateral or sitting patient positioning and avoidance of systemic opioids. There is no consensus on whether Continuous Positive Airway Pressure (CPAP), the mainstay treatment for OSA, should be administered in the event of apneas, desaturation or if hypoxia persists with supplemental oxygen. However, most institutions recommend using the patient's CPAP equipment post-operatively and also at night.

\section{PERI-OPERATIVE ANESTHESIA CONCERNS IN PREGNANT PATIENTS WITH SDB}

\section{Difficult Obstetric Airway}

The most fearful and major peri-operative concern in high risk pregnant patients undergoing general anesthesia with SDB is acute hypoxia associated with a difficult airway, difficult mask ventilation and subsequent cardiac dysrhythmias. A "difficult airway" is defined as a clinical situation in which a conventionally trained anesthesiologist experiences problems with mask ventilation, with tracheal intubation or with both [65]. Failed intubation rates in the pregnant population are $1: 240[66,67]$ compared to $1: 2330$ in the general surgical population [68]. Reasons for failure include high risk parturients e.g. preeclampsia, obesity, SDB undergoing general anesthesia, out of hours general anesthesia, less experienced staff, lack of familiarization with difficult airway equipment and poor collegial support within the operating room and from senior anesthetic staff [69]. Failure to intubate does not kill the patient, repeated attempts to intubate and failure to oxygenate will kill the patient. In this dire situation, maternal safety is the primary concern of the anesthesiologist not fetal safety.

As previously mentioned, upper airway dimensions decrease as pregnancy progresses $[70,71]$ and nasal rhinitis and congestion are commonplace symptoms [48]. Oropharyngeal size matters to the anesthesiologist. The smaller the upper airway space the greater the risk of a failed intubation. These reduced oropharyngeal dimensions may also predispose the parturient to obstructive upper airway episodes as the ability of the pharyngeal muscles to overcome negative inspiration will result in greater upper airway resistance during breathing [72]. The pregnant airway mucosa is easily traumatized and may bleed profusely. Soiling of an already difficult airway will make oral intubation extremely hard or impossible. Also, the view of direct laryngoscopy, after induction of general anesthesia may be distorted due to poor patient positioning, and poorly applied cricoid pressure [73]. A smaller endotracheal tube may be required especially if the larynx is edematous e.g. preeclampsia. Consequences of a failed or difficult intubation in parturients with SDB include pulmonary aspiration of gastric contents, esophageal intubation, bronchospasm, airway obstruction and maternal death from an inability to ventilate and more importantly oxygenate the parturient undergoing general anesthesia.

Although respiratory physiological changes in pregnancy improve maternal oxygenation at a time when oxygen consumption increases in response to the growing fetus, these changes also render the parturient especially vulnerable to general anesthesia. An elevated diaphragm caused by the growing fetus causes pulmonary compression with ventilation/perfusion mismatch. This in combination with obesity and an operative supine position with left lateral tilt, grossly reduces functional residual capacity (FRC) and minimal respiratory physiologic reserve.

As in nocturnal sleep management of SDB, optimal patient positioning is essential both prior to anesthesia induction and upon extubation, in order to improve the upper airway structure for intubation success and enable a good respiratory effort after extubation. In morbidly obese patients, Collins et al. [74] describe the ramped position as "at least a $90^{\circ}$ angle between the mandible and chest; the face higher than the chest; and external auditory meatus at the same horizontal level as the sternal angle," as optimal positioning for intubation.

On anesthetic induction, an anesthesiologist anticipates rapid oxygen desaturation. Hence time taken from induction to intubation needs to be as short as possible. Preoxygenation is an imperative step for all pregnant women prior to induction for general anesthesia. Preoxygenation is ideally 3 minutes or 8 deep breaths over one minute [75]. During pre-oxygenation, the anesthesiologist replaces nitrogen with oxygen (denitrogenation) and delays the onset of hypoxia in the event of a difficult airway. Creating an oxygen reservoir buys the anesthesiologist vital seconds for intubation.

This rapid oxygen desaturation and short-term hypoxic maternal state on induction of general anesthesia can be explained by the respiratory physiological changes in pregnancy, however a deoxygenated maternal state is further compounded by conditions that make oxygenation via mask ventilation and oral intubation difficult e.g. SDB, preeclampsia, obesity. Lung volumes as measured by spirometry, change from a non-pregnant woman to a pregnant woman. The expiratory reserve volume and residual volume comprise the FRC. The FRC represents a patient's ventilatory reservoir. It's the total volume of gas that remains in the lungs at the end of a normal tidal volume breath. The FRC reduces up to $20 \%$ by the $3^{\text {rd }}$ trimester of pregnancy. Decreases in FRC also occur with postural changes i.e. upright to supine position, obesity and restrictive lung disease. Age has no effect, however a female FRC is $10 \%$ less than that of a male [76]. Maternal ventilation in 
Table 3. Respiratory Physiological Changes in Pregnancy

\begin{tabular}{|l|}
\hline Decreased Functional Residual Capacity $(\downarrow 20 \%)$ \\
\hline Increased Minute Ventilation $(\uparrow 50 \%)$ \\
\hline Increased Oxygen Consumption $(\uparrow 20-40 \%)$ \\
\hline Decreased Chest Wall Compliance \\
\hline Increased Alveolar Ventilation $(\uparrow 50-70 \%)$ \\
\hline
\end{tabular}

pregnancy increases to over $50 \%$ of normal values in the third trimester. This increase is primarily due to an increase in tidal volume (Table 3). Further changes in maternal ventilation also occur in labor due to pain and anxiety.

During pregnancy from the first trimester onwards a woman develops a respiratory alkalosis with $\mathrm{PaCO} 2$ values of $4.0 \mathrm{KPa}$ or $31 \mathrm{mmHg}$. This change is caused by an increase in maternal progesterone levels which increase ventilation [77]. The increase in maternal cardiac output does not keep up with the increase in oxygen consumption. This leads to an increased arteriovenous oxygen difference which causes a slight fall in $\mathrm{PaO} 2$ value. As mentioned above in the supine position this decline in $\mathrm{PaO} 2$ becomes more evident and values may readily fall below $13.5 \mathrm{kPa}(100 \mathrm{mmHg})$. Once again this emphasizes the importance of maternal preoxygenation prior to anesthetic induction.

\section{Maternal and Fetal Oxygenation in an SDB Parturient}

The maternal oxygen delivery system consists of multiple components balanced to provide optimal oxygen supply to all tissues and is arranged such that an abnormality in one component is compensated by adjustments in others [78]. This principle is depicted in an oxygen dissociation curve (ODC). The ODC is a graph that shows the saturation of hemoglobin measured as a percentage at various partial pressures of oxygen. The purpose of an oxygen dissociation curve is to show the equilibrium of oxyhemoglobin and nonbonded hemoglobin at various partial pressures. As the erythrocytes release the oxygen to tissues, the partial pressure of oxygen will decrease. Consequently, the oxyhemoglobin releases the oxygen to form hemoglobin [79]. The sigmoid shape of the ODC forms due to cooperative bonding of oxygen to the four hemoglobin polypeptide chains. Cooperative bonding is the increased ability of oxygen to bind to a hemoglobin subunit that has bound oxygen. Three factors that cause a right shift in the ODC and release of oxygen to the tissues include temperature, $\mathrm{pH}$ and 2,3-diphosphogylcerate (DPG). An increase in temperature denatures the bond between oxygen and hemoglobin. A decrease in $\mathrm{pH}$ i.e. an increase in acidity by addition of carbon dioxide or other acids causes a Bohr Shift. A Bohr shift causes more oxygen to be available to tissues as oxygen tension increases [79]. Finally, an increase in 2,3-DPG, an organic phosphate that binds to hemoglobin and rearranges it's state, decreases the affinity of oxygen for hemoglobin. In normal pregnancy, P-50 values are increased hence the curve is shifted to the right and oxygen is readily uploaded to the tissues. The extent of the shift is directly related to the duration of pregnancy [80].

How does SDB affect the ODC? A study by Maillard et al. [81] compared 15 non-pregnant patients with severe sleep apnea to a group of 10 healthy subjects. Their results showed an increase in 2,3-DPG levels and a higher P-50 in the group with severe sleep apnea. These changes would cause a right shift in the ODC and protect against tissue hypoxia. However a 2008 study by Clause et al. [82] was unable to repeat these results. This group measured the ODC and 2,3 DPG concentrations in a group of 88 non-pregnant patients who presented to the sleep laboratory with a history of snoring or obstructive sleep apnea-hypopnea syndrome (OSAHS). They observed no difference between the 56 OSAHS patients and 32 non-OSAHS for the P-50 and 2,3DPG. They concluded that patients with OSAHS who are normoxemic during the day have a comparable oxyhemoglobin affinity than non-apneic subjects. Interestingly, the ODC of preeclamptic parturients shifts to the left i.e. oxygen is not readily released to the fetal or placental tissues [80]. This is an area for further study in the pregnant population with SDB.

Fetal hemoglobin comprises up to $80 \%$ of fetal blood and has a greater affinity for oxygen than adult hemoglobin. This displaces the fetal oxy-hemoglobin dissociation curve to the left. The fetus vigorously defends its oxygen supply. Studies have shown $[83,84]$ that maternal oxygen delivery to the utero-placental unit is 5-fold greater than fetal consumption and that fetal oxygen delivery and consumption are the same at low vs. high altitude i.e. maternal chronic hypoxia in vivo. Fetal oxygen delivery is unchanged despite the substantial decrease in maternal arterial oxygen tension from $12 \mathrm{kPa}$ $(90 \mathrm{mmHg})$ to $7 \mathrm{kPa}(52.5 \mathrm{mmHg})$. This study by Zamudio et al. [83] confirms that maternal oxygen delivery is not related to chronic maternal hypoxia and raises a serious question about the extent to which hypoxic episodes associated with SDB actually affect fetal oxygenation and what other mechanisms are at work. Sleep disordered breathing has been associated with increased rates of prematurity, intrauterine growth restriction, low infant Apgar scores and increased mortality $[14,85,86]$. In a later study, Zamudio et al. [87] proposed that the reduction in fetal growth in conditions of chronic hypoxia is associated with decreased delivery of glucose to the fetus and decreased fetal glucose consumption. The decrease in fetal glucose delivery is the result of increased anaerobic metabolism of glucose by the placenta. Study data support that hypoglycemia mediated fetal growth restriction is a result of increased placental metabolism of glucose.

While maternal and fetal hypoxia are not desirable states, equally, excessive fetal oxygen uptake is not ideal as fetal hyperoxia causes free radical formation which may promote tissue injury in perfusion re-perfusion injury [88]. Infant oxygen administration is associated with tissue injury in bronchopulmonary dysplasia, retinopathy of prematurity, persistent ductus arteriosus, necrotizing enterocolitis and intracranial hemorrhage [89-91].

In summary, obstetric anesthesia in patients with SDB is safe yet when maternal and fetal mortality and morbidity are affected the primary peri-operative anesthesia event usually involves difficult obstetric airway management. The difficult airway continues to be an elusive problem, with an incidence that has largely remained unchanged and with complications arising from management that remain a leading cause of anesthetic morbidity and mortality [92]. A seventeen year 
review of failed obstetric intubation revealed that this adverse event usually takes place out of hours with inexperienced staff and involves high risk patients [69]. This conclusion was also echoed in a recent national audit by the Royal College of Anaesthetists (RCoA) and Difficult Airway Society (DAS) looking prospectively at major complications of airway management throughout the UK. They identified patient, judgment, education and training, equipment and resources and communication as leading contributory factors of airway misconduct [54]. There were 4 reported obstetric patients out of 133 complicated airway management cases. The obstetric cases took place out of hours, involved high risk patients and intubation problems occurred at emergency cesarean sections.

The Centre for Maternal and Child Enquires (CMACE) 2006-2008 [54] audit recommends regular rehearsal and assessment of effective management of a failed tracheal intubation as well as better interdisciplinary communication. Yet, successful management of a difficult parturient airway is becoming a forfeited skill; with improved patient safety for cesarean section through neuraxial anesthesia, reduction in the number of general obstetric anesthetics being administered as well as a reduction in difficult obstetric airway drills being carried out following a difficult airway algorithm [93]. Training and education are key factors in the recognition of pregnancy related co-morbidities e.g. SDB, obesity, preeclampsia associated with a difficult airway. Fortunately, anesthesia adverse events are rare representing only $1.5 \%$ of all maternal deaths, yet the consequences are devastating.

\section{Treatment of SDB Patients in Pregnancy with Nasal CPAP}

Nasal CPAP has been used to treat pregnant patients with SDB with good maternal outcomes [94, 95]. A number of small studies have also shown that nocturnal nasal CPAP reduces hypertension in preeclamptics, [95, 96]. Better patient education on sleep habits e.g. adopt lateral position, sit upright should also be implemented. As standard of care, an anesthesiology consult should also be initiated in high risk obstetric patients with SDB to allow for pre-operative evaluation and peripartum assessment.

\section{Anesthetic Management Strategies for SDB Parturients}

\section{Pre-Operative Evaluation}

The obstetric anesthesiologist needs to develop a heightened state awareness of SDB in a parturient whose body habitus and airway are in a transient state of flux. Signs and symptoms of SDB should become apparent from the second trimester onwards. The anesthesiologist needs to take a salient sleep history and examine the patient for predisposing signs of SDB such as obesity, neck circumference, facial swelling and tonsillar hypertrophy. Optimize patient care by ensuring appropriate management of SDB as well as associated conditions e.g. pregnancyrelated hypertension, preeclampsia, diabetes and obesity.

Discussion of the different types of anesthesia available to the patient is important in order to allay patient anxiety and promote realistic patient expectations. Explanation of the possible outcomes from a GA in a patient with symptomatic SDB i.e. supplemental post-operative oxygen, overnight monitoring with ECG and pulse oximetry, prolonged intubation and transfer to Intensive Care Unit for emergence should be a standard of care. The anesthesiologist needs to be proactive in informing the patient and promoting realistic patient expectations; as well as communicating with the obstetric team, discussing anesthetic management with the obstetrician and sharing any patient concerns.

If SDB is suspected, inform the patient, obstetrician and nursing staff. Acquire a formal diagnosis from a sleep clinic if there is time and treat with nasal CPAP. If that is not possible consider guidance from the ASA, OSA practice guideline recommendations and tailor your anesthetic management accordingly i.e. avoid GA if possible, implement early neuraxial anesthesia if indicated, promote nocturnal positioning in bed i.e. semi-recumbent, avoid use of narcotics and institute nocturnal monitoring if necessary.

\section{Peri-Operative Strategies}

The anesthesiologist needs to proactively seek out and discount or suspect SDB in pregnancy. Validated OSA screening questionnaires and algorithms may be considered yet none to date have been validated in the obstetric population. Implementation of certain pre-operative OSA screening tools e.g. Berlin questionnaire, ESS questionnaire is not appropriate to detect sleep apnea in high risk obstetric patients [19].

The anesthesiologist in conjunction with the obstetrician should ensure that the patient receives appropriate monitoring in the right setting, appropriate treatment e.g. nasal CPAP and sleep toileting advice. Ensure that your facility has the equipment and personnel to manage a difficult airway, deliver supplemental oxygen, ventilate postoperatively, analyze arterial blood gases, take a chest X-ray, and monitor and treat hypoventilation and hypoxic episodes post operatively. The difficult airway cart needs to be routinely checked and easily located.

Does the patient have a previous history of difficult intubation and is there a difficult airway note in her file? A junior anesthesiologist should always call for senior assistance if concerned about a difficult obstetric airway. On a practical note, should the patient require operative intervention under general anesthesia during her pregnancy, consider neuraxial anesthesia if relevant and analgesic management: regional anesthesia vs.GA; non-steroidal antiinflammatory medication vs. opioids.

On oral intubation the direct laryngoscopy (DL) view of the larynx should be noted. If a patient has a challenging airway, describe the reasons for difficulty in the notes and explain to the patient post-operatively. Also be aware that the difficult parturient airway may return to normal airway status in the post-partum period. Preparation and knowledge are key to pre-emptively avoid an airway disaster.

Only extubate once the patient is fully conscious and has control of airway reflexes, Consideration of patient positioning post-operatively; semi recumbent or sat upright and avoidance of the supine position is imperative. Respiratory complications are most common and will most likely occur a few hours after surgery [72]. Overnight monitoring should be performed to monitor for any respiratory and/or cardiac events. 


\section{SUMMARY}

Recent ASA OSA practice guidelines, subsequent development of OSA algorithms and validated pre-operative OSA screening questionnaires in the general surgical population have been developed to counteract the impact of peri-operative complications associated with OSA patients requiring anesthesia. Anesthesiologists are now being able to recognize the condition, request referral for investigation as well as tailor their anesthetic to reduce adverse, perioperative anesthetic related sequelae. The 2006 ASA recommendations based on expert opinion and studies in the general population; do not appear to be detrimental to any patient undergoing general anesthesia and should be considered when appropriate in the absence of evidence based clinical management of SDB in the obstetric population.

Management of SDB in pregnancy requires an understanding of the normal physiological changes in pregnancy as well as early detection of associated high risk comorbidities. Interdisciplinary communication and vigilance by all team members are principles that should be routinely applied on all labor and delivery suites for a successful maternal and fetal outcome. SDB in the obstetric population is increasingly being highlighted in both academic and medical arenas and will hopefully promote further reduction in maternal and fetal morbidity and mortality.

Despite a dramatic fall in obstetric anesthetic complications [97] there is no role for complacency in obstetric anesthesia with the continued change in obstetric patient demographics e.g. $>35$ yo, increased obesity, and complex medical conditions. Education and awareness of anesthesiology management of sleep disordered breathing, preeclampsia, pregnancy-related hypertensive disorders and obesity that adversely affect the maternal airway and promote peri-operative respiratory and cardiovascular events are also imperative. Management of the SDB parturient is an obstacle that can be readily tackled if well prepared, well trained, and when team communication is optimal. Further research into SDB and peri-operative anesthesia management is urgently required to determine the best evidence based practice in the obstetric population

\section{REFERENCES}

[1] Young T, Palta M, Dempsey J, Skatrud J, Weber S, Badr S. The occurrence of sleep disordered breathing among middle aged adults. N Engl J Med 1993; 328: 1230-5

[2] Redline S, Kump K, Tishler PV, Browner I, Ferette V. Gender differences in sleep disordered breathing in a community-based sample. Am J Respir Crit Care Med 1994; 149: 722-6

[3] Bixler EO, Vgontzas AN, Lin HM, et al. Prevalence of sleepdisordered breathing in women: effects of gender. Am J Respir Crit Care Med 2001; 163: 608-13

[4] Schoenfield A, Ovadia Y, Neri A, Freedman S. Obstructive sleep apnea (OSA)-implications in maternal-fetal medicine. A hypothesis. Med Hypotheses 1989; 30: 51-4

[5] Louis JM, Auckley D, Sokol RJ, Mercer BM. Maternal and neonatal morbidities associated with obstructive sleep apnea complicating pregnancy. Am J Obstet Gynecol 2010; 202: 261, e15 .

[6] Chen YH, Kang JH, Lin CC, Wang IT, Keller JJ, Lin HC. Obstructive sleep apnea and the risk of adverse pregnancy outcomes. Am J Obstet Gynecol 2012; 206: 136.e1-5 xx-xx.
[14] Franklin KA, Holmgren PA, Jönsson F, Poromaa N, Stenlund H, Svanborg E. Snoring, pregnancy induced hypertension and growth retardation of the fetus. Chest.2000; 117:137-41.

[15] O'Brien LM, Bullough AS, Owusu JT, et al. Pregnancy-onset habitual snoring, gestational hypertension, and preeclampsia: prospective cohort study. Am J Obstet Gynecol 2012; 207(6): 487.e1-9.

[16] Hawkins JL, Arens JF, Bucklin BA, et al. Practice Guidelines for Obstetric Anesthesia. Anesthesiology 2007; 106: 843-63.

[17] Chung F, Yegneswaran B, Liao P, et al. Validation of the Berlin Questionnaire and American Society of Anesthesiologists Checklist as screening tools for obstructive sleep apnea in surgical patients. Anesthesiology 2008; 108: 822-30.

[18] Ramachandran SK, Josephs LA. A meta-analysis of clinical screening tests for obstructive sleep apnea. Anesthesiology 2009; 110: 928-39.

[19] Facco FL, Ouyang DW, Zee PC, Grobman WA. Development of pregnancy-specific screening tool for sleep apnea. J Clin Sleep Med 2012 15; 8: 389-94.

[20] O'Brien LM, Bullough AS, Shelgikar AV, Chames MC, Armitage R, Chervin RD. Validation of Watch-PAT-200 against polysomnography during pregnancy. J Clin Sleep Med 2012; 8: 287-94.

[21] Ruehland WR, Rochford PO, O'Donoghue FJ, Pierce RJ, Singh P, Thornton AT. The New AASM Criteria for Scoring Hypopneas: Impact on the Apnea Hypopnea Index. Sleep 2009; 3: 150-7.

[22] American Academy of Sleep Medicine Task Force. Sleep-related breathing disorders in adults: recommendations for syndrome definition and measurement techniques in clinical research. Sleep 1999; 22: 667-89.

[23] Kim JA, Lee JJ. Preoperative predictors of difficult intubation in patients with obstructive sleep apnea syndrome. Can J Anesth 2006; 53:4 393-7.

[24] Gyulay S, Olson LG, Hensley MJ, King MT, Allen KM, Saunders NA.A comparison of clinical assessment and home oximetry in the diagnosis of obstructive sleep apnea. Am Rev Respir Dis 1993; 147; 50-3.

[25] Kryger M, Roth T, Dement W. Principles and practice of sleep medicine. $3^{\text {rd }}$ ed. Philadelphia, PA Saunders 2000; p. 827.

[26] Boutonnet M, Faitot V, Katz A, Salomon L, Keita H. Mallampati class changes during pregnancy, labor and after delivery: can these be predicted? Br J Anaesth 2010; 104: 67-70.

[27] Kodali BS, Chandrasekhar S, Bulich LN, Topulos GP, Datta S. Airway changes during labor and delivery. Anesthesiology 2008; 108: 357-62.

[28] Guilleminault C, Dement W. Sleep Apnea syndromes and related disorders. In: Williams R, Karacen I, Moore C, Eds. Sleep disorders: diagnosis and treatment. New York: Wiley 1988: pp. 4771.

[29] Bliwise D, Feldman D, Bliwise N, et al. Risk factors for sleep disordered breathing in heterogeneous geriatric populations. J Am Geriatr Soc 1987; 35: 132-41. 
[30] Vgontas A, Tan T, Bixler E, Martin L, Shubert D, Kales A. Sleep apnea and sleep disruption in obese patients. Arch Intern Med 1994; 154: 1705-11.

[31] Kristensen MS. Airway management and morbid obesity. Eur J Anaesthesiol 2010; 27: 923-7.

[32] Harris AT, Morell D, Bajaj Y, Martin-Hirsch DP. A discussion of airway and respiratory complications along with general considerations in obese patients. Int J Clin Pract 2010; 64: 802-6.

[33] Endler GC, Mariona FG, Solol RJ et al. Anesthesia related mortality in Michigan 1972-84. Am J Obstet Gynecol 1988; 159: 187-93.

[34] The AANA Foundation Closed Malpractice Claims Study Obstetric Anesthesia. AANA J 2002; 70: 97-104.

[35] Loredo JS, Ancoli-Israel S, Dimsdale JE. Sleep quality and blood pressure dipping in obstructive sleep apnea. Am J Hypertens 2001; 14: 887-92.

[36] Redman CW, Beilin LJ, Bonnar J. Reversed diurnal blood pressure rhythm in hypertensive pregnancies. Clin Sci Mol Med Suppl 1976; 3: 687S-9S.

[37] Beilin LJ, Deacon J, Michael CA, et al. Diurnal rhythms of blood pressure, plasma renin activity, angiotensin II and catecholamines in normotensive and hypertensive pregnancies. Clin Exp Hypertens B 1983; 2: 271-93.

[38] Izci B, Riha RL, Martin SE, et al. The upper airway in pregnancy and pre-eclampsia. Am J Respir Crit Care Med 2003; 167:137-40

[39] Chung F, Yegneswaran B, Herrera F, Shenderey A, Shapiro CM. Patients with difficult intubation may need referral to sleep clinics. Anesth Analg 2008; 107: 915-20.

[40] Payen JF, Jaber S, Levy P, Pepin JL, Fischler M. Obstructive sleepapnoea syndrome in adult and its peri-operative management. Ann Fr Anesth Reanim 2010; 29: 787-92.

[41] Hiremath AS, Hillman DR, James AL, Noffsinger WJ, Platt PR, Singer SL. Relationship between difficult tracheal intubation and obstructive sleep apnoea. Br J Anaesth 1998; 80: 606-11.

[42] Siyam MA, Benhamou D. Difficult endotracheal intubation in patients with Sleep Apnea Syndrome. Anesth Analg 2002; 95: 1098-102.

[43] Morgan BM, Magni V, Goroszenuik T. Anesthesia for emergency cesarean section. BJOG 1990; 97: 420-4.

[44] Young T, Finn L, Palta M. Chronic nasal congestion at night is a risk factor for snoring in a population based cohort study. Arch Intern Med 2001; 161: 1514-9.

[45] White DP, Douglas NJ, Pickett CK, Weil JV, Zwillich CW. Sexual influence on the control of breathing. J Appl Physiol 1983; 54:8749.

[46] Yannone ME, McCurdy JR, Goldfien A. Plasma progesterone levels in normal pregnancy, labor and the puerperium. II. Clinical data. Am J Obstet Gynecol 1968; 101: 1058-61.

[47] Elkus R, Popovich J Jr. Respiratory physiology in pregnancy. Clin Chest Med 1992; 13: 555-65.

[48] Mabry RL. Rhinitis of pregnancy. South Med J 1986; 79: 965-71.

[49] Young T, Finn L, Kim H. Nasal obstruction as a risk factor for sleep disordered breathing. The University of Wisconsin Sleep and Respiratory Research Group. J Allergy Clin Immunol 1997; 99: S757-62.

[50] Venkata C, Venkateshiah SB. Sleep-disordered breathing during pregnancy. J Am Board Fam Med 2009; 22: 158-68.

[51] Eastwood PR, Platt PR, Shepherd K, Maddison K, Hillman DR. Collapsibility of the upper airway at different concentrations of propofol anesthesia. Anaesthesiology 2005; 103: 470-7.

[52] Drummond GB. Effects of sufentanil and morphine on ventilation. Br J Anaesth 1995; 74: 492-3.

[53] Warwick JP, Mason DG. Obstructive syndrome in children. Anaesth 1998; 53: 571-9.

[54] Rennotte MT, Baele P, Aubert G, Rodenstein DO. Nasal continuous positive airway pressure in the peri-operative management of patients with obstructive sleep apnea submitted to surgery. Chest 1995; 107: 367-74.

[55] Centre for Maternal and Child Enquires (CMACE), Centre for Maternal and Child Enquiries (CMACE). Saving Mothers' Lives: reviewing maternal deaths to make motherhood safer- 2006- 2008. The Eighth Report on Confidential Enquiries into Maternal Deaths in the United Kingdom. BJOG 2011; 118: 1-203.

[56] Major complications of airway management in the UK: results of the Fourth National Audit Project of the Royal College of
Anaesthetists and the Difficult Airway Society. Part 1: Anaesthesia. Br J Anaesth 2011; 106: 617-31.

[57] Cullen DJ. Obstructive sleep apnea and postoperative analgesia - A potentially dangerous combination. J Clin Anesth 2001; 13: 83- 5

[58] Katsiris S, Williams S, Leighton BL, Halpern S. Respiratory arrest following intrathecal injection of sufentanil and bupivacaine in a parturient . Can J Anaesth 1998; 45: 880-3.

[59] Jaffee JB, Drease GE, Kelly T, Newman LM. Severe respiratory depression in the obstetric patient after intrathecal meperidine or sufentanil. Int J Obstet Anest 1997; 6: 182-84.

[60] Lu JK, Manullang, TR, Staples MH, Kern SE, Bailey PL. Maternal Respiratory Arrests, Severe Hypotension and Fetal Distress after Administration of Intrathecal, Sufentanil, and Bupivacaine after Intravenous Fentanyl. Anesthesiology 1997; 87: 170-2.

[61] Kato R, Shimamoto H, Terui K, Yokota K, Miyao H. Delayed respiratory depression associated with $0.15 \mathrm{mg}$ intrathecal morphine for cesarean section: a review of 1915 cases. J Anesth 2008; 22: 112-6.

[62] Horlocker TT, Burton AW, Connis RT, et al. Practice guidelines for the prevention, detection and management of respiratory depression associated with neuraxial opioid administration Anesthesiology 2009; 110: 1-1.

[63] Gali B, Whalen FX Jr, Gay PC, et al. Management plan to reduce risks in peri-operative care of patients with presumed obstructive sleep apnea syndrome. J Clin Sleep Med 2007; 3: 582-8.

[64] Hwang D, Shakir N, Limann B, et al. Association of SleepDisordered Breathing with postoperative complications. Chest 2008; 133; 1128-34.

[65] Practice guidelines for management of the difficult airway. A report by the American Society of Anesthesiologists Task Force on Management of the Difficult Airway. Anesthesiology 1993; 78: 597-602.

[66] Barnardo PD, Jenkins JG. Failed tracheal intubation in obstetrics: a 6 year review in a UK region. Anesthesiology 2000; 55: 685-94.

[67] Rahman K, Jenkins JG. Failed tracheal intubation in obstetrics: no more frequent but still managed badly. Anesthesiology 2005; 60: 168-71.

[68] Samsoon GL, Young JR. Difficult tracheal intubation: A retrospective study. Anesthesiology 1987; 42: 487-90.

[69] Hawthorne L, Wilson R, Lyons G, Dresner M. Failed intubation revisited: 17-yr experience in a teaching maternity unit. $\mathrm{Br}$ Anesthesiology 1996; 76: 680-4

[70] Pilkington S, Carli F, Dakin MJ, et al. Increase in Mallampati score during pregnancy. Br J Anesthesiology 1995; 74: 638-42.

[71] Izci B, Vennelle M, Liston WA, Dundas KC, Calder AA Douglas NJ. Sleep Disordered breathing and upper airway size inpregnancy and post-partum. Eur Respir J 2006: 27(2): 321-7.

[72] Chung SA, Yuan H, Chung FA systemic Review of Obstructive Sleep Apnea and its implications for Anesthesiologists. Anesth Analg 2008; 107:1543-63.

[73] Smith KJ, Dobranowski, J, Yip, G, Dauphin, A, Choi PT Cricoid pressure displaces the esophagus: an observational study using magnetic resonance imaging. Anesthesiology 2003; 99: 60-4

[74] Collins JS, Lemmens HJ, Brodsky JB, Brock-Utne JG, Levitan RM. Laryngoscopy and morbid obesity: a comparison of the "sniff" and "ramped" positions. Obes Surg 2004; 14:1171-75

[75] Baraka AS, Taha SK, Aouad MT, El-Khatib MF, Kawkabani NI Preoxygenation: comparison of maximal breathing and tidal volume breathing techniques. Anesthesiology 1999; 91: 612-6

[76] Nunn's Applied Respiratory Physiology. Andrew Lumb: Churchill Livingstone 2010.

[77] Lim VS, Katz AI, Lindheimer MD. Acid-base regulation in pregnancy. Am J Physiol 1976; 231:1764-9.

[78] Woodson RD. Physiological significance of oxygen dissociation curve shifts. Crit Care Med. 1979; 7:368-73

[79] Animal Physiology: Adaptation and Environment. Knut SchmidtNielsen. Cambridge University Press: UK 1997.

[80] Kambam JR, Handte RE, Brown WU, Smith BE. Effect of normaland pre-eclamptic pregnancies on the oxygen dissociation curve. Anesthesiology 1986; 65: 426-7.

[81] Maillard D, Fleury B, Housset B, Laffont S, Chabolle J, Derenne JP. Decreased oxyhemoglobin affinity in patients with sleep apnea syndrome. Am Rev Respir Dis 1991; 143: 486-9

[82] Clause D, Detry B, Rodenstein D, Liistro G. Stability of oxyhemoglobin affinity in patients with obstructive sleep apnea- 
hypopnea syndrome without daytime hypoxemia. J Appl Physiol 2008; 105: 1809-12

[83] Zamudio S, Postigo L, Illsley NP, et al. Maternal oxygen delivery is not related to altitude and ancestry-associated differences in fetal growth. J Physiol 2007; 582: 883-95

[84] Postigo L, Heredia G, Illsley NP, et al. Where the O2 goes to: preservation of human fetal oxygen delivery and consumption at high altitude. J Physiol 2009; 587: 693-708

[85] Izci-Balserak B, Pien GW. Sleep-disordered breathing and pregnancy: potential mechanisms and evidence for maternal and fetal morbidity. Curr Opin Pulm Med 2010; 16: 574-82

[86] Louis JM, Auckley D, Sokol RJ, Mercer BM. Maternal and neonatal morbidities associated with obstructive sleep apnea complicating pregnancy. Am J Obstet Gynecol 2010; 202: 261.e1-5

[87] Zamudio S, Torricos T, Fik E, et al. Hypoglycemia and the origin of hypoxia-induced reduction in human fetal growth 2010; 5: e8551, 1-10.

[88] Khaw KS, Wang CC, Ngan Kee WD, Pang CP, Rogers MS. Effects of high inspired oxygen fraction during elective Caesarean section under spinal anaesthesia on maternal and fetal oxygenation and lipid peroxidation. Br J Anaesth 2002; 88: 18-23.

[89] Clyman RI, Saugstad OD, Mauray F. Reactive oxygen metabolites relax the lamb ductus arteriosus by stimulating prostaglandin production. Circ Res 1989; 64: 1-8
[90] Northway WH, Rosan RC, Porter DY. Pulmonary disease following respiratory therapy of hyalinemembrane disease. Bronchopulmonary dysplasia. N Engl J Med 1967; 276: 357-68

[91] Campbell K. Intensive oxygen therapy as a possible cause of retrolental fibroplasia: a clinical approach. Med J Aust 1950; 2: 4850

[92] Peterson GN, Domino KB, Caplan RA, Posner KL, Lee LA, Cheney FW. Management of the difficult airway: a closed claims analysis. Anesthesiology 2005; 103: 33-9

[93] Bullough AS, Carraretto M. A United Kingdom national obstetric intubation equipment survey. Int J Obstet Anesth 2009; 18: 342-5.

[94] Guilleminaiult C, Kreutzer M, Chang JL. Pregnancy, sleep disordered breathing and treatment with nasal continuous positive airway pressure. Sleep Med 2004; 5: 43-51.

[95] Poyares D, Guilleminault C, Hachul H, et al. Pre-eclampsia and nasal CPAP: part 2. Hypertension during pregnancy, chronic snoring, and early nasal CPAP intervention. Sleep Med 2007; 9: $15-21$

[96] Edwards N, Blyton DM, Kirjavainen T, Kesby GJ, Sullivan CE. Nasal pressure positive airway pressure reduces sleep-induced blood pressure increments in preeclampsia. Am J Respir Crit Care Med 2000; 162: 252-7

[97] Hawkins JL, Chang J, Palmer SK, Gibbs CP, Callaghan WM. Anesthesia-Related Maternal Mortality in the United States: 19792002. Obstet Gynecol 2011; 117: 69-7. 